\title{
AKUPUNKTUR, AURIKULOMEDIZIN UND LASERTHERAPIE 2018
}

\section{Liebe Kolleginnen und Kollegen,}

die Fachgebiete unserer Zeitschrift gewinnen immer mehr an Akzeptanz, weil wir durch vermehrte Forschung immer bessere Kenntnisse über Prävention, Diagnose und Therapie erhalten. Dennoch bedarf es ständig weiterer innovativer Forschungsprojekte, um die neuen Verfahren in eine allgemein anerkannte Praxis zu transferieren oder, falls sie der wissenschaftlichen Prüfung nicht standhalten, eben eliminieren zu können. Sicher wird auch das Jahr 2018 groBe Fortschritte bringen, und es gibt zahlreiche Bereiche, von denen wir hoffen, dass sie noch mehr Akzeptanz finden.

Akupunktur für Schmerzen ist wahrscheinlich das am meisten erforschte Gebiet.Wir sind zuversichtlich, dass die Forschung auch in diesem Jahr zu diesem Themenfokus nicht nachlassen wird. Akupunktur für die psychische Gesundheit ist weniger erforscht. Dennoch, viele Menschen sind auf Medikamente angewiesen, die wiederum zahlreiche Nebenwirkungen haben. In diesem Bereich wird es für die Akupunktur, Aurikulomedizin und Lasertherapie noch viel zu forschen geben. Für 2018 sind sowohl in Asien als auch in den USA und in Europa größere Forschungsarbeiten zur Thematik Angst und Depression geplant. Auch die WHO (World Health Organization) empfiehlt inzwischen den Einsatz der Akupunktur und spricht mittlerweile ja auch nicht mehr nur von traditioneller und komplementärer Medizin, sondern von gezielter Förderung der integrativen Medizin, die ja auch die Lasertherapie beinhaltet.
Forschungsthemata wie rheumatoide Arthritis und Endometriose sind andere Zielrichtungen. Rheumatoide Arthritis braucht definitiv bessere Behandlungsansätze, Akupunktur und Lasertherapie können Schübe und Schmerzen reduzieren. Vielfach wird dies mit Kräutern, Diät- und geänderten Lebensstilansätzen kombiniert.

Es gibt aber auch einige, in diesem Jahr hochrangig publizierte akupunkturkritische Studien, die nicht den für die Akupunkturbefürworter gewünschten Erfolg gezeigt haben (Smith CA et al. Effect of acupuncture vs sham acupuncture on live births among woman undergoing in vitro fertilization - a randomized clinical trial,JAMA 2018, Impact Faktor: 44.4). Diese Resultate sind aus unserer Sicht unbedingt zu berücksichtigen, da Therapien bei speziellen PatientInnengruppen nur dann angewendet werden sollen, wenn deren wissenschaftliche Evidenz auch gesichert ist.

Laserspezifisch liefert das Jahr 2018 viele Highlights. So zum Beispiel wird vom 3. bis 6. Oktober $2018 \mathrm{im}$ französischen Nizza der WALT (World Association for Laser Therapy) Kongress stattfinden. Ein Workshop und eine spezielle Sitzung werden sich speziell mit der Thematik Laserakupunktur beschäftigen. Selbstverständlich gibt es Ende des Jahres auch wieder die ISLA-Asien-Konferenz in Bangkok (siehe ISLA-Vorschau).

Wir würden uns freuen, Sie persönlich begrüßen zu dürfen. Es warten spannende Programme auf Sie.

Viel Freude beim Lesen dieser Ausgabe!

Mit freundlichen Grüßen
Michael Weber

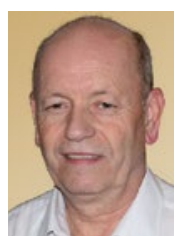

Dr. med. Dipl. Chem. Michael Weber

President for Medical and Clinical Applications der International Society for Medical Laser Applications

(ISLAtranscontinental)

Klinik Neu-Mariahilf, Humboldtallee 10-12,

D-37073 Göttingen, Tel. +49 5273/367 780

E-Mail:info@isla-laser.org,Internet:www.isla-laser.org
Gerhard Litscher

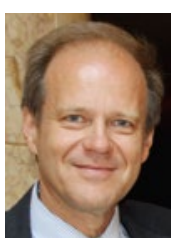

Univ.-Prof. Prof. h.c. DI Dr. techn.

Dr. scient. med. Gerhard Litscher

President for Science and Research der International Society

for Medical Laser Applications (ISLAtranscontinental)

Medizinische Universität Graz, Auenbruggerplatz 29,

A-8036 Graz, Tel: +43 316/385-13907, -83907

E-Mail:gerhard.litscher@medunigraz.at,Internet:http://litscher.info 